\title{
KLK6 and KLKI 3 predict tumor recurrence in epithelial ovarian carcinoma
}

\author{
NMA White', M Mathews ${ }^{2}$, GM Yousef ${ }^{3}$, A Prizada ${ }^{4}$, C Popadiuk ${ }^{5}$ and JJE Doré*,I \\ 'Division of BioMedical Sciences, Memorial University, St John's, Newfoundland, Canada; '2Division of Community Health and Humanities, Memorial \\ University, St John's, Newfoundland, Canada; ${ }^{3}$ Department of Laboratory Medicine, The Keenan Research Centre, The Li Ka Shing Knowledge Institute, \\ St Michael's Hospital, Toronto, Ontario, Canada; ${ }^{4}$ Discipline of Laboratory Medicine, Memorial University, St John's, Newfoundland, Canada; 'Discipline of \\ Obstetrics and Gynecology, Health Sciences Centre, St John's, Newfoundland, Canada
}

BACKGROUND: The human kallikrein-related peptidase family consists of 15 genes. Twelve of these genes are overexpressed in ovarian cancer and may represent potential markers for diagnosis, prognosis, and/or response to treatment. The aim of this study was to determine the prognostic significance of kallikrein-related peptidase 6 (KLK6) and kallikrein-related peptidase 13 (KLK/3) in epithelial ovarian cancer by quantifying gene expression levels with tumour pathology and patient survival data.

METHODS: Total RNA was isolated from 106 patients diagnosed with primary ovarian cancer, as well as 8 normal ovary controls. Samples were analysed by quantitative real-time PCR for $K L K 6$ and $K L K / 3$ expression. Correlation between kallikrein gene expression and clinical characteristics was evaluated with the $\chi^{2}$-test. Survival analysis was performed using Kaplan-Meier and Cox proportional hazards regression models.

RESULTS: Expression levels of both KLK6 and KLKI 3 mRNA were significantly increased in invasive cancers relative to normal ovaries $(P=0.002$ and 0.039 respectively). High $K L K 6$ and $K L K 13$ expression was an indicator of poor prognosis, with patients having a shorter recurrence-free survival $(P=0.002$ and 0.027 respectively). High KLK6 expression was also significantly associated with lower overall survival $(P=0.0 \mathrm{II})$. When subjected to multivariate analysis, patients with either high KLK6 or KLKI 3 were 3- and 2.2-fold, respectively, more likely to have a recurrence than patients with low kallikrein expression.

CONCLUSION: These data show increased mRNA expression of KLK6 and KLKI 3 in ovarian cancer compared to normal ovarian tissues. High KLK6 or KLKI 3 expression in primary ovarian tumours can significantly predict prognosis in terms of recurrence-free survival and overall survival. In all, this study shows KLK6 and KLKI3 as potential biomarkers and may be therapeutic targets for treatment of ovarian cancer.

British Journal of Cancer (2009) I 01, I I07- | | I3. doi:I0.1038/sj.bjc.6605280 www.bjcancer.com

Published online 25 August 2009

(c) 2009 Cancer Research UK

Keywords: human kallikrein-related peptidase 6; human kallikrein-related peptidase 13; ovarian cancer; ovarian carcinogenesis; biomarkers

Ovarian carcinoma, known as 'the disease that whispers,' is the most lethal of all the gynaecological malignancies. Annually, approximately 23000 new cases and 14000 deaths in the United States are due to ovarian cancer (Holschneider and Berek, 2000). This malignancy presents a great clinical challenge as it is often diagnosed in the late stages due to its anatomical location and relative asymptomatic occurrence (Holschneider and Berek, 2000). Approximately $75 \%$ of patients are diagnosed in late stage disease (stage III/IV) and have a 5-year survival rate of only $15-20 \%$, compared to a $80-90 \%$ 5-year survival rate when diagnosed in the early stages (stage I/II; Schink, 1999). The disease is treatable and in most cases curable if diagnosed in the early stages.

*Correspondence: Dr JJE Doré, Division of BioMedical Sciences, Memorial University, Room 53।4, 300 Prince Phillip Drive, St John's, Newfoundland, AIC 3V6 Canada; E-mail: jdore@mun.ca

Received 6 April 2009; revised 27 July 2009; accepted 29 July 2009; published online 25 August 2009
Recently, a family of serine proteases has been identified on human chromosome $19 \mathrm{q} 13$ and named the human kallikreinrelated peptidase family (KLK; Yousef and Diamandis, 2003). The family consists of 15 genes, of which 12 (KLK2, KLK3, KLK4, KLK5, KLK6, KLK7, KLK8, KLK10, KLK11, KLK13, KLK14, and KLK15) appear to be overexpressed in ovarian cancer. As been shown for prostate-specific antigen (PSA/KLK3) in prostate cancer, these may represent potential novel biomarkers for ovarian carcinoma (Borgono and Diamandis, 2004). This study focuses on two members of the kallikrein-related peptidase family, KLK6 and KLK13.

Kallikrein-related peptidase $6(K L K 6)$ was initially identified by three different groups who named it protease $M$ in breast cancer (Anisowicz et al, 1996), zyme in Alzheimer's disease (Little et al, 1997), and neurosin in colon adenocarcinoma (Yamashiro et al, 1997). KLK6 is reported to have differential expression in ovarian, breast, uterine, and colon cancers (Anisowicz et al, 1996; Tanimoto et al, 2001; Hoffman et al, 2002; Ogawa et al, 2005; Santin et al, 2005). KLK6 is overexpressed at both the gene and protein levels in ovarian cancer and has been associated with poor patient 
prognosis (Diamandis et al, 2000a; Tanimoto et al, 2001; Kountourakis et al, 2008). Recently, KLK6 has been implicated in the loss of cell-cell contact and promotion of cell proliferation, migration, and invasion in keratinocytes (Klucky et al, 2007). With the involvement in these biological functions, the overexpression of KLK6 in ovarian cancer suggests it may be involved in promoting cancer invasion and metastasis. In vitro assays have shown that recombinant KLK6 proteins are capable of extracellular matrix (ECM) protein digestion and neutralising KLK6 antibodies can decrease the rate of migration of ovarian cancer cell lines, further supporting this hypothesis (Ghosh et al, 2004).

Kallikrein-related peptidase 13 (KLK13) was first identified as downregulated in breast cancer tissues and cell lines (Yousef et al, 2000 ). However, $50 \%$ of malignant ovarian tissues had increased KLK13 expression relative to nearly undetectable levels in normal or benign tissue (Kapadia et al, 2003). In addition, Scorilas et al (2004) found high levels of KLK13 in early stage cancers and consequently associated high KLK13 with a better prognosis. Similar to KLK6, KLK13 can degrade major components of the ECM and when treated with an anti-KLK13 antibody, an ovarian cancer cell line showed decreased migratory capacity (Kapadia et $a l, 2004)$. On the basis of these previously observed KLK6 and KLK13 effects on ovarian cancer cells, the aim of this study was to evaluate the prognostic significance of KLK6 and KLK13 in epithelial ovarian cancer by quantifying gene expression levels and correlating them with clinical variables and patient survival data.

\section{MATERIALS AND METHODS}

\section{Ovarian cancer samples}

The study analysed formalin-fixed, paraffin-embedded ovarian tissues from 106 cases of sporadic ovarian carcinoma diagnosed in the province of Newfoundland and Labrador, Canada between 1983 and 2002. Eight normal ovary samples were also obtained for comparison. Tissues were collected from pathology archives and selected based on hematoxylin and eosin stains reviewed by a pathologist. Clinical staging was performed using the standard International Federation of Gynecology and Obstetrics staging, with tumours graded as borderline, well differentiated (grade I), moderately differentiated (grade II), or poorly differentiated (grade III). Clinical history was obtained by review of patients' medical records in accordance with Memorial University's human investigation committee protocol.

Medical treatment of patients consisted of a total abdominal hysterectomy, bilateral salpingo-oophorectomy, omentectomy, and tumour staging. When cancer was not in stage $1 \mathrm{~A}$ grade 1 or borderline, patients went on to receive chemotherapy. As the scope of this study spans from 1983 to 2002, chemotherapy regimes changed over this time. Before 1995/96 patients received cisplatin and cyclophosphamide, given for 6-9 cycles, at the discretion of the physician. After 1995/96 with the introduction of taxanes, treatments involved the combining of taxol with either cisplatin or carboplatin. A small number of patients with late stage (3C and 4) disease before 1998 were also treated with chemotherapy as a neoadjuvant pre-surgery.

\section{Immunohistochemistry}

Sections were cut $4 \mu \mathrm{m}$ thick and dried on glass slides overnight. Sections were deparaffinised in xylene and re-hydrated through decreasing graded alcohols. Antigen retrieval was performed using a pressure cooker for $10 \mathrm{~min}$ in citrate buffer ( $\mathrm{pH}$ 6.0). Slides were incubated overnight in primary antibody, washed twice with phosphate-buffered saline $(\mathrm{pH}$ 7.4) followed by either of two detection methods. Detection of KLK6 immunocomplex was carried out using Envision (Dako, Mississauga, Ontario, Canada), whereas localisation of KLK13 immunocomplex was performed with $\mathrm{LSAB}+$, Link, and streptavidin reagents (Dako). Immune complexes were visualised by incubating with diaminobenzidine and sections were counterstained with hematoxylin. Immunolocalisation of KLK6 was carried out using a rabbit polyclonal antibody (Diamandis et al, 2000b), whereas KLK13 localisation was carried out using a mouse monoclonal antibody, clone 33.1 (Kapadia et al, 2003).

\section{Quantitative real-time PCR}

Five $10 \mu \mathrm{M}$ sections were cut from paraffin-embedded tissues for nucleic acid isolation. Total RNA was extracted using the High Pure RNA Paraffin kit (Roche, Indianapolis, IN, USA) according to the manufacturer's protocol. Total RNA concentration was determined spectrophotometrically at $260 \mathrm{nM}$ and samples were stored at $-80^{\circ} \mathrm{C}$. The presence of high molecular weight total RNA was determined using a NanoDrop 1000 Spectrophotometer (NanoDrop Technologies Inc., Wilmington, DE, USA) and ethidium bromide staining of samples using formaldehyde gel electrophoresis. cDNA was synthesised using $2 \mu \mathrm{g}$ total RNA and Superscript First-Strand Synthesis System for RT-PCR (Invitrogen, Carlsbad, CA, USA) with random hexamers to ensure representation of all $\mathrm{mRNA}$ independent of polyadenylated tail.

Quantitative real-time polymerase chain reaction (qRT-PCR) was performed using the ABI Prism 7000 (Applied Biosystems, Foster City, CA, USA). Primer/probe sets were purchased as premade TaqMan Assays on Demand for KLK13, KLK6, and glyceraldehyde 3-phosphate dehydrogenase (GAPDH; described in Supplementary Table 1). Thermal cycling conditions were according to the manufacture's protocol and all reactions were performed in triplicate. Relative quantification was defined as the amount of the specific mRNA normalised to a normal ovary as determined using the comparative cycle threshold $\left(C_{\mathrm{T}}\right)$ method. The relative target gene expression was defined as $2^{-\Delta \Delta \mathrm{CT}}$, where $\Delta \Delta C_{\mathrm{T}}=\Delta C_{\mathrm{T}}$ normal ovary $-\Delta C_{\mathrm{T}}$ ovarian cancer. A normal ovarian sample having the median level of expression for a specific target gene was chosen as the calibrator sample and used to normalise expression of all other samples (i.e. target gene expression $=1$ ). $\Delta C_{\mathrm{T}}$ is defined as $C_{\mathrm{T}}$ target $-C_{\mathrm{T} G A P D H}$, where the target genes were KLK13 and KLK6. Relative to the calibrator sample, target gene relative expression (RE) was classified as being low or high kallikrein expression. The expression level for determining high expressing samples was defined as one standard deviation above the mean value for all normal ovarian RE, for each specific target gene. Samples with an RE equal to or above this value were classified as high expression, whereas samples below the cut-off were classified as low expression. The cut-off for KLK6 was determined as $5.211 \mathrm{RE}$ and KLK13 as $0.981 \mathrm{RE}$. Using a standard deviation above the normal mean $\mathrm{RE}$ of each target gene allowed us to utilise the inherent variation of each target gene expression to independently determine its cut-off value.

\section{Statistical analysis}

All statistical analysis was performed with the SPSS statistical package for PC (version 13.0; SPSS Inc., Chicago, IL, USA). The relationships between KLK6 and KLK13 mRNA expression and patient clinical characteristics were analysed with a $\chi^{2}$-test. For survival analysis, two end points were examined; cancer recurrence (defined as either a local recurrence or metastasis) and death. These end points were used to calculate the recurrence-free survival (RFS) and overall survival (OS) respectively. Recurrencefree survival is defined as the time from first diagnosis to the time of first detected recurrence or metastasis. Overall survival is defined as the time from initial diagnosis to the time of death. Two survival models, the Kaplan-Meier and the Cox proportional hazard regression, were used for the analysis. The Kaplan-Meier 
model was used to examine survival between the patients expressing kallikreins at low or high levels, whereas significance was measured with the log-rank test. The Cox proportional hazard regression model, using both univariate and multivariate models, was used to determine the hazard ratio.

\section{RESULTS}

\section{Immunohistochemical localisation of KLK6 and KLK13 in} ovarian carcinoma

Figure 1 illustrates the pattern of KLK6 and KLK13 localisation in ovarian surface epithelium (OSE) and epithelial ovarian carcinoma. Both KLK6 and KLK13 were localised in the cytoplasm of the normal ovarian surface epithelial (Figures 1A and B). Both KLK6 and KLK13 showed staining in all types of ovarian adenocarcinoma. Shown here is strong staining for both KLKs in serous adenocarcinoma (Figures 1C and D), with KLK6 shown expressed in a mucinous adenocarcinoma (Figure 1E) and KLK13 expression in a clear cell tumour (Figure 1F). In all cases, staining was most prominent in epithelial cells, whether they were normal surface epithelium or carcinoma. Immunohistological evaluation of protein levels resulted in no significant associations with clinical characteristics. The intensity of staining may not be only because of the variation of kallikrein expression in each pathological specimen, but also be contributed by the variations in fixing and embedding procedures used during the processing of tissues. To quantify the differences in expression more accurately, we extracted total RNA from ovarian samples and analysed by qRT-PCR, comparing KLK expression in ovarian tumours to normal samples.

\section{Association between Kallikrein gene expression and clinical variables}

The relationship between KLK6 and KLK13 expression levels and clinical characteristics is summarised in Table 1. Patient ages range from 20 to 89 years with a mean age of 60 years. Compared to patients with low $K L K 6$, a significantly larger proportion of patients with high KLK6 had invasive cancer $(P=0.002)$ and late stage cancers $(P=0.001)$. Compared to patients with low $K L K 13$, a larger proportion of patients with high $K L K 13$ had invasive cancer $(P=0.039)$. Unlike $K L K 6, K L K 13$ was not associated with clinical stage.

At diagnosis, $65 \%$ of all patients had serous ovarian cancer. In supplementary analysis, when we compared serous to non-serous ovarian cancers (mucinous, endometrioid, clear cell, and unknown), high KLK6 expression was associated with serous carcinomas $(P=0.001$; data not shown). Interestingly, all endometrioid ovarian cancers had high KLK13 expression relative to normal ovaries, but the small sample size was unable to provide sufficient power for a conclusive association.

\section{KLK6 expression association with recurrence and survival}

Kaplan-Meier survival curves indicated patients with high KLK6 expression were more likely to have a shorter RFS $(P=0.002$, Figure $2 \mathrm{~A})$ and $\mathrm{OS}(P=0.011$, Figure $2 \mathrm{~B})$, when compared to

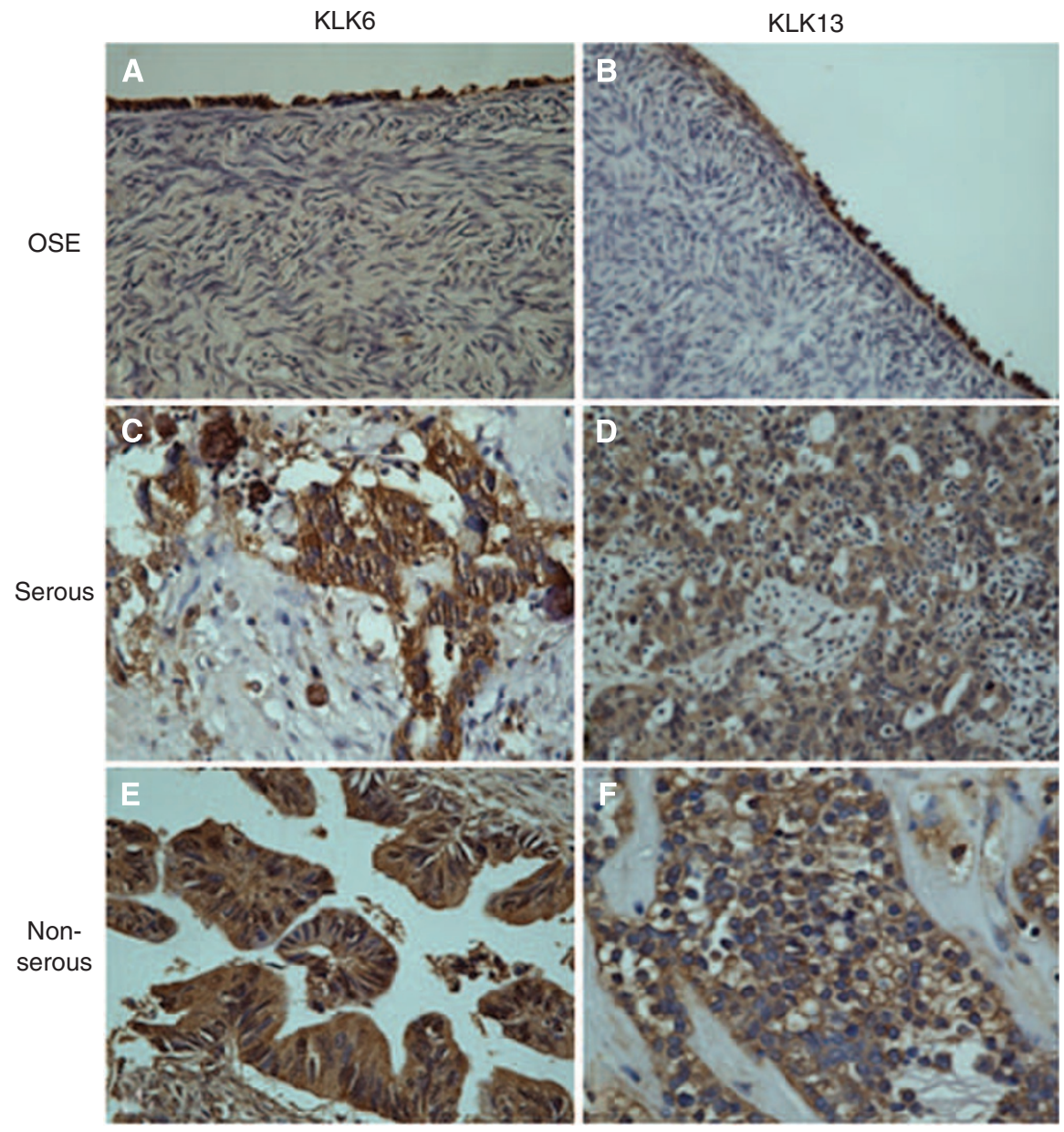

Figure I KLK6 and KLKI3 expression in normal ovarian surface epithelium (OSE) and epithelial ovarian cancer. The normal OSE stains positive for both KLK6 (A) and KLKI3 (B). Serous epithelial cancers express KLK6 (C) and KLKI 3 (D) in the cytoplasm. In a mucinous ovarian tumour, KLK6 shows strong expression (E). KLKI3 is positively expressed in a clear cell ovarian tumour $(\mathbf{F})$. All photomicrographs were taken at $\times 40$ magnification. 
Table I Association between KLK6 and KLKI3 mRNA expression with clinicopathological data for 106 ovarian carcinoma patients and 8 normal ovaries

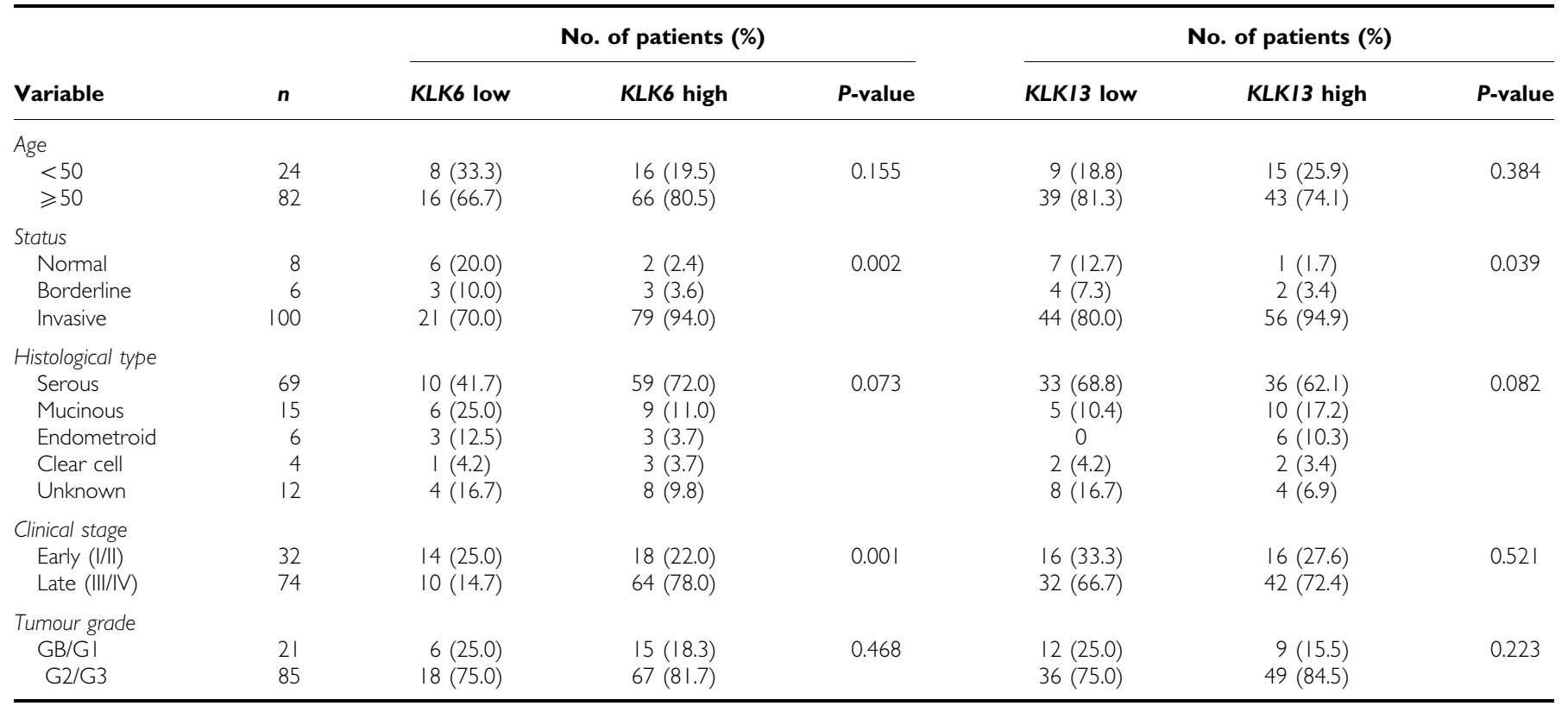

A

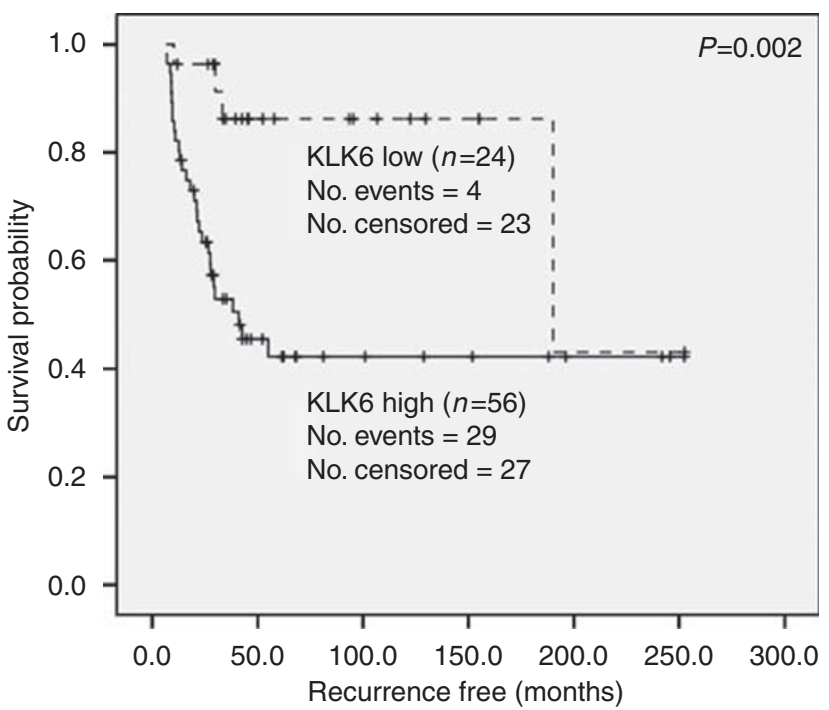

B

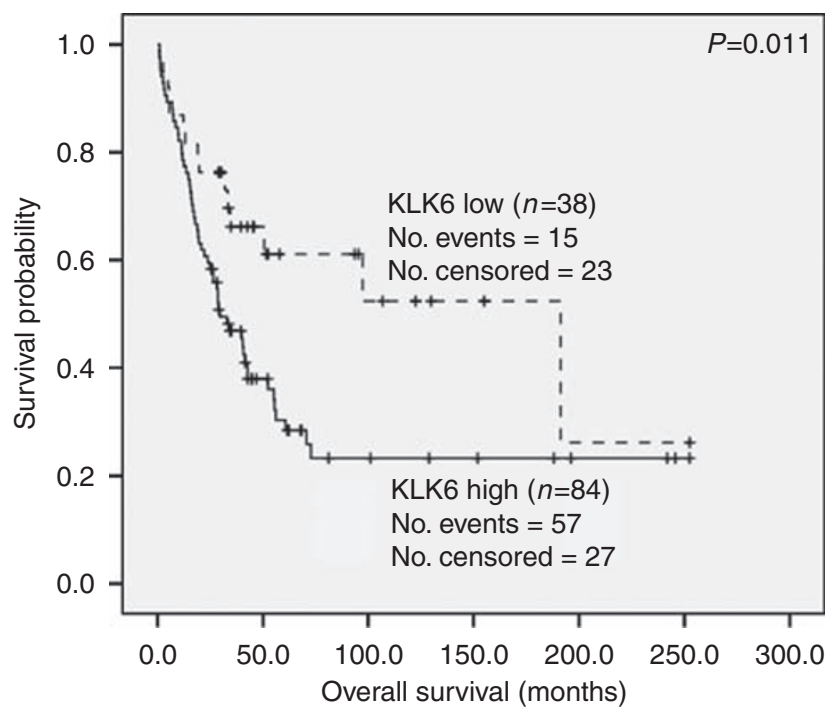

Figure 2 Kaplan-Meier survival curves showing the proportion of patients who are recurrence free $(\mathbf{A})$ and alive $(\mathbf{B})$ at the given time after diagnosis. Patients are stratified based on KLK6 expression and compared by the log-rank test. Patients whose tumours are KLK6 low are represented with the broken line, whereas patients whose tumours are KLK6 high are represented by the solid line. n, number of patients.

patients with low KLK6 expression. These data are further supported by the Cox regression analysis presented in Table 2 . In univariate analysis, patients with high KLK6 expression had a greater risk of recurrence $(P=0.004)$ than patients with low KLK6 expressing tumours. As expected, clinical stage $(P<0.001)$, tumour grade $(P=0.012)$, and histological type $(P=0.024)$ were all significant predictors of recurrence. In the multivariate model (Table 3), high KLK6 expression remains significant as a predictor of recurrence $(P=0.040)$, indicating these patients are approximately three times more likely to have a recurrence than patients with low KLK6 expression. Overall, late clinical stage (stage III/IV) was the strongest predictor of recurrence $(P=0.001)$.

When OS is examined in a Cox univariate model (Table 2), high KLK6 expression is significantly associated with a shorter OS $(P=0.013)$. As expected, clinical stage $(P<0.001)$, tumour grade $(P<0.001)$, and histological type $(P=0.037)$ are all associated with a shorter OS. Interestingly, patients 50 years of age or older, at the time of diagnosis, also had a significantly shorter OS $(P=0.045)$ than patients under the age of 50 . When these factors were included in a multivariate analysis (Table 3), clinical stage was the strongest predictor of OS, patients with late stage (stage III/IV) cancers had a four-fold increased likelihood of a shorter OS $(P=0.001)$ than patients with early stage (Stage I/II) cancer. Other clinical characteristics lost their predictive significance of OS when subjected to multivariate analysis, including high $K L K 6$ expression $(P=0.215)$.

\section{High $K L K 13$ expression in ovarian tumours is associated with poor prognosis}

When KLK13 expression was analysed with the Kaplan-Meier model, patients with high KLK13 expression had a shorter RFS than patients with low KLK13 expression $(P=0.027$; Figure $3 \mathrm{~A})$. 
Table 2 Univariate Cox regression analysis of $K L K 6, K L K / 3$, and other clinicopathological variables

\begin{tabular}{|c|c|c|c|c|c|c|}
\hline \multirow[b]{2}{*}{ Variable } & \multicolumn{3}{|c|}{ RFS } & \multicolumn{3}{|c|}{ os } \\
\hline & HR & $95 \% \mathrm{Cl}$ & $P$-value & HR & $95 \% \mathrm{Cl}$ & $P$-value \\
\hline \multicolumn{7}{|l|}{ Univariate analysis } \\
\hline $\begin{array}{l}\text { KLKO } \\
\text { Low }\end{array}$ & 1.00 & - & - & 1.00 & - & - \\
\hline High & 4.59 & $1.61-13.08$ & 0.004 & 2.06 & $1.16-3.63$ & 0.013 \\
\hline \multicolumn{7}{|l|}{ KLKI3 } \\
\hline Clinical stage ${ }^{a}$ (ordinal) & 11.89 & $3.61-39.15$ & $<0.001$ & 5.91 & $2.82-12.35$ & $<0.001$ \\
\hline Tumour grade ${ }^{b}$ (ordinal) & 3.42 & $1.31-8.94$ & 0.012 & 3.83 & $1.82-8.07$ & $<0.001$ \\
\hline Histological type ${ }^{c}$ (ordinal) & 2.31 & $1.12-4.78$ & 0.024 & 1.67 & $|.03-2.7|$ & 0.037 \\
\hline Age $^{\mathrm{d}}$ (ordinal) & 2.76 & $0.97-7.87$ & 0.057 & 1.98 & $1.02-3.87$ & 0.045 \\
\hline
\end{tabular}

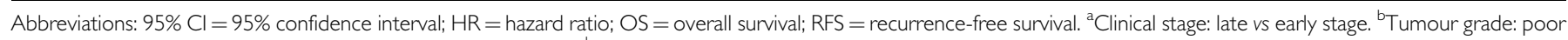
vs well differentiated. 'Histological type : serous vs non-serous. ${ }^{\mathrm{d}} \mathrm{Age:} \geqslant 50$ vs $<50$.

Table 3 Multivariate Cox regression analysis of KLK6 expression

\begin{tabular}{|c|c|c|c|c|c|c|}
\hline \multirow[b]{2}{*}{ Variable } & \multicolumn{3}{|c|}{ RFS } & \multicolumn{3}{|c|}{ os } \\
\hline & HR & $95 \% \mathrm{Cl}$ & $P$-value & HR & $95 \% \mathrm{Cl}$ & $P$-value \\
\hline \multicolumn{7}{|l|}{ Multivariate analysis } \\
\hline Low & 1.00 & - & - & 1.00 & - & - \\
\hline High & 3.03 & $1.05-8.74$ & 0.040 & 1.45 & $0.81-2.60$ & 0.215 \\
\hline Clinical stage ${ }^{\text {a }}$ (ordinal) & 8.57 & $2.45-30.05$ & 0.001 & 3.73 & $1.70-8.18$ & 0.001 \\
\hline Age $^{d}$ (ordinal) & 2.19 & $0.72-6.67$ & 0.167 & 1.47 & $0.74-2.94$ & 0.271 \\
\hline
\end{tabular}

Abbreviations: $95 \% \mathrm{Cl}=95 \%$ confidence interval; $\mathrm{HR}=$ hazard ratio; OS = overall survival; RFS = recurrence-free survival. ${ }^{\mathrm{a} C l i n i c a l}$ stage: late vs early stage. ${ }^{\text {b}}$ Tumour grade: poor vs well differentiated. 'Histological type : serous vs non-serous. ${ }^{\mathrm{d}} \mathrm{Age:} \geqslant 50$ vs $<50$.

A

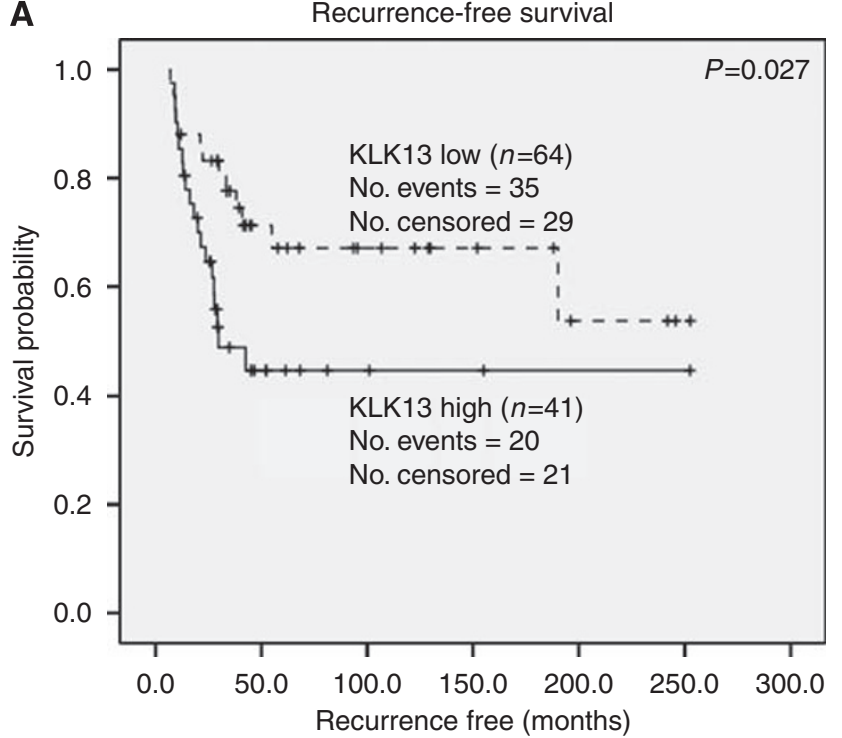

B

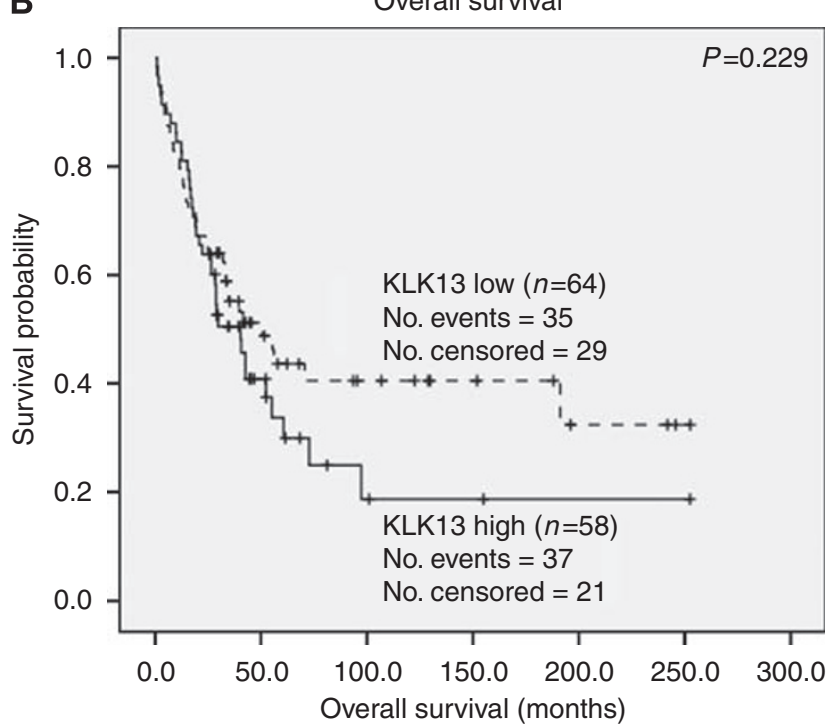

Figure 3 Kaplan-Meier survival curves showing the proportion of patients who are recurrence free $(\mathbf{A})$ and alive $(\mathbf{B})$ at the given time following diagnosis. Patients are stratified based on KLKI 3 expression and compared by the log-rank test. Patients whose tumours are KLKI 3 low are represented with the broken line, whereas patients whose tumours are KLKI3 high are represented by the solid line. $n$, number of patients.

The strength of association between KLK13 high expressing tumours and survival outcome is presented in a Cox regression model (Tables 3 and 4). Univariate analysis showed high KLK13 as a significant predictor of recurrence $(P=0.030)$, indicating a 2.2 fold increased probability of recurrence compared to low KLK13 (Table 3). When KLK13 expression was examined in a multivariate model, it retains the ability to significantly predict a shorter RFS ( $P=0.047$; Table 4). Late clinical stage and serous type cancer also significantly predict a shorter RFS $(P=0.001$ and 0.024 respectively). When we examine KLK13 expression along with other factors in a multivariate model, late clinical stage was the only significant predictor of OS $(P<0.001)$. 
Table 4 Multivariate Cox regression analysis of KLKI3 expression

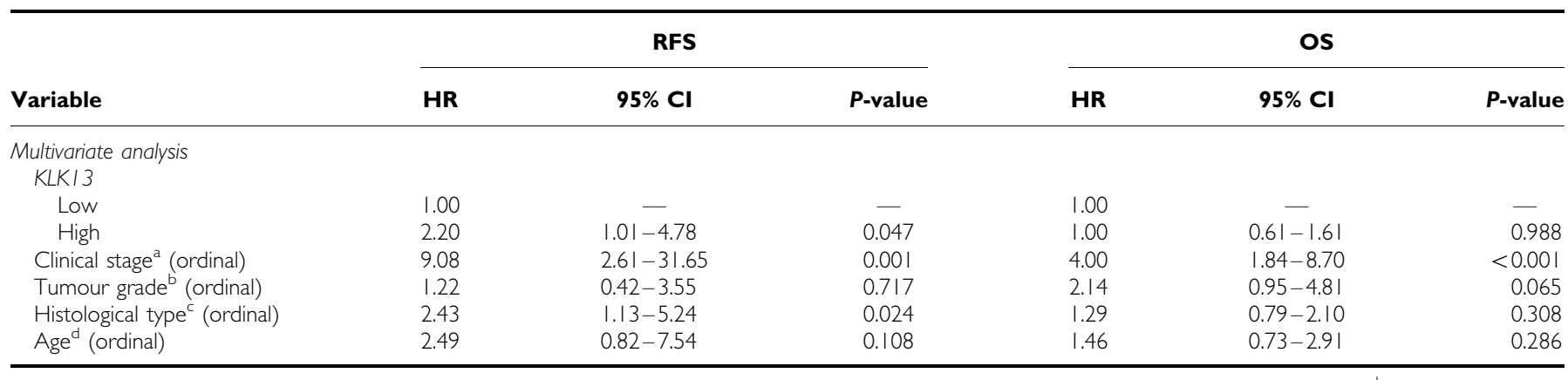

Abbreviations: $95 \% \mathrm{Cl}=95 \%$ confidence interval; $\mathrm{HR}=$ hazard ratio; $\mathrm{OS}=$ overall survival; $\mathrm{RFS}=$ recurrence-free survival. ${ }^{\mathrm{a}} \mathrm{Clinical}$ stage: late vs early stage. ${ }^{\mathrm{b}}$ Tumour grade: poor vs well differentiated. 'Histological type : serous vs non-serous. ${ }^{\mathrm{d}}$ Age: $\geqslant 50$ vs $<50$.

\section{DISCUSSION}

Unlike other reproductive malignancies, such as prostate cancer, ovarian cancer lacks a biomarker that may be used for screening. Currently, CA125, the only marker used in ovarian cancer patients, is reliable only for monitoring response to treatment and disease recurrence. The identification of early biomarkers for ovarian cancer may lead to novel therapeutic applications and potential screening tests. This study was aimed at examining the expression of KLK6 and KLK13 in ovarian cancer to determine their diagnostic or prognostic value.

When we examined kallikrein protein expression by immunohistochemistry (Figure 1), we found that although there appeared to be increased KLK6 and KLK13 expression in ovarian cancer tumours relative to normal OSE, there were no significant associations to clinical variables or survival. These results are similar to previous studies that found increased expression of KLK6 in primary pancreatic ductal adenocarcinoma (Ruckert et al, 2008) and salivary gland tumours (Darling et al, 2006), but found no significant association with survival. Kallikreins 6 and 13 are expressed in normal epithelium (Petraki et al, 2001, 2003), including the OSE as we have shown here. Ovarian cancer is thought to arise from either OSE or OSE cells bordering inclusion cysts (Kaku et al, 2003), therefore it is expected that ovarian cancer cells would express a basal level of kallikrein expression. Given the semi-quantitative nature of immunohistochemistry and the fact that KLKs are secreted proteins, to see a significant difference in the cellular levels between normal OSE and ovarian cancer would require not only changes in KLK protein production, but also a significant change in the rate of kallikrein exocytosis.

With these limitations in quantifying protein expression, we used qRT-PCR to assess kallikrein mRNA expression in ovarian cancer tissues. High KLK6 mRNA expression was associated with the presence of serous ovarian cancer and late stage disease. These results are similar to previous studies, which found increased KLK6 expression in ovarian cancers when compared to normal ovarian tissue (Anisowicz et al, 1996; Tanimoto et al, 2001; Ni et al, 2004). Interestingly, previous studies have also associated high KLK6 serum levels with advanced ovarian cancer (stage III/IV) and serous tumour histology (Hoffman et al, 2002; Shan et al, 2007). Ovarian serous tumours of borderline and low grade are thought to arise from a step-wise progression from adenoma to borderline tumour to carcinoma through the Ras-Raf signalling pathway (Bell, 2005). Recently, KLK6 expression and secretion has been shown to be Ras dependent in a colon carcinoma cell line (Henkhaus et al, 2008). Constitutively active mutant K-Ras resulted in enhanced colon cancer cell invasion through both laminin and Matrigel matrixes. Together, these data suggest that Ras-Raf mutations may increase the invasive potential of these borderline tumours through increased expression of KLK6. This may also hold true for advanced ovarian cancers, as we show high KLK6 expression is associated with later stage, more invasive, cancers. High-grade serous carcinomas have a notably different pathogenesis than lowgrade serous tumours. High-grade serous tumours commonly have mutations in p53, BRCA 1, and/or BRCA2 (Christie and Oehler, 2006). To date there have be no reports indicating a relationship between KLK6 and p53 or BRCA genes. When we looked at only high- and low-grade serous tumours, we found no significant differences in KLK6 expression $(P=0.498)$. Despite the apparent differences in the molecular oncogenesis of low- and high-grade serous tumours, their upregulation of KLK6 suggests a common pathway is activated in both types of tumours.

Our study is the first to report KLK13 mRNA expression in normal ovary and ovarian cancer patients. The expression of KLK13 mRNA in normal OSE was extremely low, whereas $55 \%$ ovarian cancers examined had high KLK13 expression. These findings support Kapadia et al (2003), who found serum levels of KLK13 were below levels of detection in healthy individuals, yet $50 \%$ ovarian cancer patients were positive for KLK13 (Kapadia et al, 2003). Interestingly, $100 \%$ of our endometrioid cancer samples were high grade and expressed KLK13 at a high level. Although our sample size is small (six cases), this expression pattern warrants further investigation. Low-grade endometrioid carcinomas have been suggested to arise from endometriosis or borderline endometrioid tumours (Obata et al, 1998), whereas high-grade endometrioid carcinomas have changes similar to high-grade serous carcinomas (Bell, 2005; Giordano et al, 2008; Press et al, 2008) suggesting endometrioid cancers may represent two separate malignancies. This characteristic expression pattern of KLK13 may represent not only a novel marker to distinguish between high- and low-grade endometrioid cancers, but a unique pathway in which KLK13 may be involved in ovarian carcinogenesis.

Since histological types of ovarian cancers present as a spectrum of pathologically and histologically different phenotypes and may represent cancers of different origins (Bell, 2005), the possibility that a panel of markers, rather than a single marker, may improve the sensitivity and specificity of detecting ovarian cancer at an early stage while it is treatable. Zheng et al (2007) found that a group of kallikrein-related peptidases, including KLK6 and KLK13, in multi-parametric combinations with other biomarkers and clinical variables can significantly predict prognosis and response to treatment in ovarian cancer patients. A similar model, including a number of kallikrein-related peptidases, has been proposed for non-small-cell lung carcinoma (Planque et al, 2008). This study confirms both KLK6 and KLK13 are overexpressed in ovarian cancer and are useful predictors of poor prognosis in ovarian cancer patients. This study is the first to report upregulation of KLK13 mRNA in ovarian cancer patients and indicated that KLK13 may represent a specific marker for endometrioid carcinoma. These findings support the potential role as kallikrein-related peptidases 6 and 13 as novel ovarian cancer biomarkers and may, in the future, offer targets for therapeutic applications. 


\section{ACKNOWLEDGEMENTS}

We thank Dr Eleftherios Diamandis (Department of Pathology and Laboratory Medicine, Mount Sinai Hospital, Toronto, Ontario, Canada) for the use of KLK6 and KLK13 antibodies. This work was supported in part by Canadian Institutes of Health Research

\section{REFERENCES}

Anisowicz A, Sotiropoulou G, Stenman G, Mok SC, Sager R (1996) A novel protease homolog differentially expressed in breast and ovarian cancer. Mol Med 2: 624-636

Bell DA (2005) Origins and molecular pathology of ovarian cancer. Mod Pathol 18: S19-S32

Borgono CA, Diamandis EP (2004) The emerging roles of human tissue kallikreins in cancer. Nat Rev Cancer 4: 876-890

Christie M, Oehler MK (2006) Molecular pathology of epithelial ovarian cancer. J Br Menopause Soc 12: $57-63$

Darling MR, Jackson-Boeters L, Daley TD, Diamandis EP (2006) Human kallikrein 6 expression in salivary gland tumors. J Histochem Cytochem 54: $337-342$

Diamandis EP, Yousef GM, Soosaipillai AR, Bunting P (2000a) Human kallikrein 6 (zyme/protease M/neurosin): a new serum biomarker of ovarian carcinoma. Clin Biochem 33: 579-583

Diamandis EP, Yousef GM, Soosaipillai AR, Grass L, Porter A, Little S, Sotiropoulou G (2000b) Immunofluorometric assay of human kallikrein 6 (zyme/protease M/neurosin) and preliminary clinical applications. Clin Biochem 33: $369-375$

Ghosh MC, Grass L, Soosaipillai A, Sotiropoulou G, Diamandis EP (2004) Human kallikrein 6 degrades extracellular matrix proteins and may enhance the metastatic potential of tumour cells. Tumour Biol 25: $193-199$

Giordano G, Azzoni C, D’Adda T, Rocco A, Gnetti L, Froio E, Merisio C, Melpignano M (2008) Human papilloma virus (HPV) status, p16(INK4a), and p53 overexpression in epithelial malignant and borderline ovarian neoplasms. Pathol Res Pract 204: $163-174$

Henkhaus RS, Gerner EW, Ignatenko NA (2008) Kallikrein 6 is a mediator of K-RAS-dependent migration of colon carcinoma cells. Biol Chem 389: $757-764$

Hoffman BR, Katsaros D, Scorilas A, Diamandis P, Fracchioli S, Rigault de la Longrais IA, Colgan T, Puopolo M, Giardina G, Massobrio M, Diamandis EP (2002) Immunofluorometric quantitation and histochemical localisation of kallikrein 6 protein in ovarian cancer tissue: a new independent unfavourable prognostic biomarker. $\mathrm{Br} J$ Cancer 87: $763-771$

Holschneider CH, Berek JS (2000) Ovarian cancer: epidemiology, biology, and prognostic factors. Semin Surg Oncol 19: 3-10

Kaku T, Ogawa S, Kawano Y, Ohishi Y, Kobayashi H, Hirakawa T, Nakano $\mathrm{H}$ (2003) Histological classification of ovarian cancer. Med Electron Microsc 36: 9-17

Kapadia C, Chang A, Sotiropoulou G, Yousef GM, Grass L, Soosaipillai A, Xing X, Howarth DH, Diamandis EP (2003) Human kallikrein 13: production and purification of recombinant protein and monoclonal and polyclonal antibodies, and development of a sensitive and specific immunofluorometric assay. Clin Chem 49: 77-86

Kapadia C, Ghosh MC, Grass L, Diamandis EP (2004) Human kallikrein 13 involvement in extracellular matrix degradation. Biochem Biophys Res Commun 323: $1084-1090$

Klucky B, Mueller R, Vogt I, Teurich S, Hartenstein B, Breuhahn K, Flechtenmacher C, Angel P, Hess J (2007) Kallikrein 6 induces Ecadherin shedding and promotes cell proliferation, migration, and invasion. Cancer Res 67: 8198-8206

Kountourakis P, Psyrri A, Scorilas A, Camp R, Markakis S, Kowalski D, Diamandis EP, Dimopoulos MA (2008) Prognostic value of kallikreinrelated peptidase 6 protein expression levels in advanced ovarian cancer evaluated by automated quantitative analysis (AQUA). Cancer Sci 99: $2224-2229$

Little SP, Dixon EP, Norris F, Buckley W, Becker GW, Johnson M, Dobbins JR, Wyrick T, Miller JR, MacKellar W, Hepburn D, Corvalan J, McClure D, Liu X, Stephenson D, Clemens J, Johnstone EM (1997) Zyme, a novel and potentially amyloidogenic enzyme cDNA isolated from Alzheimer's disease brain. J Biol Chem 272: 25135-25142
(CIHR), grant no. 62277, and the Faculty of Medicine, Memorial University, St John's, Newfoundland, Canada.

Supplementary Information accompanies the paper on British Journal of Cancer website (http://www.nature.com/bjc)

Ni X, Zhang W, Huang KC, Wang Y, Ng SK, Mok SC, Berkowitz RS, Ng SW (2004) Characterisation of human kallikrein 6/protease $M$ expression in ovarian cancer. $\mathrm{Br}$ J Cancer 91: $725-731$

Obata K, Morland SJ, Watson RH, Hitchcock A, Chenevix-Trench G, Thomas EJ, Campbell IG (1998) Frequent PTEN/MMAC mutations in endometrioid but not serous or mucinous epithelial ovarian tumors. Cancer Res 58: 2095 - 2097

Ogawa K, Utsunomiya T, Mimori K, Tanaka F, Inoue H, Nagahara $H$, Murayama S, Mori M (2005) Clinical significance of human kallikrein gene 6 messenger RNA expression in colorectal cancer. Clin Cancer Res 11: $2889-2893$

Petraki CD, Karavana VN, Diamandis EP (2003) Human kallikrein 13 expression in normal tissues: an immunohistochemical study. J Histochem Cytochem 51: $493-501$

Petraki CD, Karavana VN, Skoufogiannis PT, Little SP, Howarth DJ, Yousef GM, Diamandis EP (2001) The spectrum of human kallikrein 6 (zyme/ protease $M /$ neurosin) expression in human tissues as assessed by immunohistochemistry. J Histochem Cytochem 49: 1431 - 1441

Planque C, Li L, Zheng Y, Soosaipillai A, Reckamp K, Chia D, Diamandis EP, Goodglick L (2008) A multiparametric serum kallikrein panel for diagnosis of non-small cell lung carcinoma. Clin Cancer Res 14: $1355-1362$

Press JZ, De Luca A, Boyd N, Young S, Troussard A, Ridge Y, Kaurah P, Kalloger SE, Blood KA, Smith M, Spellman PT, Wang Y, Miller DM, Horsman D, Faham M, Gilks CB, Gray J, Huntsman DG (2008) Ovarian carcinomas with genetic and epigenetic BRCA1 loss have distinct molecular abnormalities. BMC Cancer 8: 17-29

Ruckert F, Hennig M, Petraki CD, Wehrum D, Distler M, Denz A, Schroder M, Dawelbait G, Kalthoff H, Saeger HD, Diamandis EP, Pilarsky C, Grutzmann R (2008) Co-expression of KLK6 and KLK10 as prognostic factors for survival in pancreatic ductal adenocarcinoma. Br J Cancer 99: $1484-1492$

Santin AD, Diamandis EP, Bellone S, Soosaipillai A, Cane S, Palmieri M, Burnett A, Roman JJ, Pecorelli S (2005) Human kallikrein 6: a new potential serum biomarker for uterine serous papillary cancer. Clin Cancer Res 11: 3320 - 3325

Schink JC (1999) Current initial therapy of stage III and IV ovarian cancer: challenges for managed care. Semin Oncol 26: 2-7

Scorilas A, Borgono CA, Harbeck N, Dorn J, Schmalfeldt B, Schmitt M, Diamandis EP (2004) Human kallikrein 13 protein in ovarian cancer cytosols: a new favorable prognostic marker. J Clin Oncol 22: $678-685$

Shan SJ, Scorilas A, Katsaros D, Diamandis EP (2007) Transcriptional upregulation of human tissue kallikrein 6 in ovarian cancer: clinical and mechanistic aspects. Br J Cancer 96: $362-372$

Tanimoto H, Underwood LJ, Shigemasa K, Parmley TH, O’Brien TJ (2001) Increased expression of protease $\mathrm{M}$ in ovarian tumors. Tumour Biol 22: $11-18$

Yamashiro K, Tsuruoka N, Kodama S, Tsujimoto M, Yamamura Y, Tanaka T, Nakazato H, Yamaguchi N (1997) Molecular cloning of a novel trypsin-like serine protease (neurosin) preferentially expressed in brain. Biochim Biophys Acta 1350: $11-14$

Yousef G, Diamandis EP (2003) Human kallikreins: common structural features, sequence analysis and evolution. Curr Genomics 4: $147-165$

Yousef GM, Chang A, Diamandis EP (2000) Identification and characterization of KLK-L4, a new kallikrein-like gene that appears to be down-regulated in breast cancer tissues. J Biol Chem 275: $11891-11898$

Zheng Y, Katsaros D, Shan SJ, de la Longrais IR, Porpiglia M, Scorilas A, Kim NW, Wolfert RL, Simon I, Li L, Feng Z, Diamandis EP (2007) A multiparametric panel for ovarian cancer diagnosis, prognosis, and response to chemotherapy. Clin Cancer Res 13: 6984-6992 